\title{
MENISCUS OF A MAGNETIC FLUID IN THE FIELD OF A CURRENT-CARRYING WIRE: TWO-DIMENSIONAL NUMERICAL SIMULATIONS
}

\author{
P.-B. Eißmann, A. Lange, S. Odenbach \\ TU Dresden, Institute of Fluid Mechanics, Chair of Magnetofluiddynamics, \\ 01062 Dresden, Germany
}

Two-dimensional calculations of the meniscus of a magnetic fluid around a currentcarrying vertical and cylindrical wire are presented. The influence of the viscosity, the angle of contact at the wire and at the rim of the vessel are studied. Based on the material properties of an experimentally used magnetic fluid, the numerically determined meniscus is compared with the experimentally measured one. The comparison is made for a linear law of magnetization as well as for the experimentally measured nonlinear magnetization curve.

1. Introduction. One of the most fascinating features of the physics of magnetic fluids (MFs) is the fact that the form and appearance of their free surface can be drastically modified under the influence of external magnetic fields. The Rosensweig instability ([1] and references therein) can be considered as an icon of such a behaviour, but also singular drops [2] or sculptures [3] fashioned from these liquids exhibit in magnetic fields an astonishing variety and beauty in observable patterns.

An apparently much simpler setup to study is the meniscus of a horizontal layer of magnetic fluid in the field of a vertical current-carrying cylindrical wire. An early analysis of that setup was given in [4], where the influence of the surface tension was ignored. More than two decades later, the renewed interest started with a numerical work [5] on the resulting nonlinear differential equation describing the evolution of the surface $\xi(r)$ as a function of the distance $r$ from the wire and taking the surface tension into account. Recently, experimental data has been published [6], which allows a comparison with the calculated results. Therefore, the aim of this paper is to present the numerical simulation of the underlying NavierStokes equation for an axially symmetrical, i.e. a two-dimensional configuration and contrast the results with the experimental data.

2. Governing equations. A picture of the meniscus around a vertical cylindrical wire is givenin the top part of Fig. 1, where the bottom part contains a sketch of the geometry. The theoretical description of the shape of the meniscus is based on the Bernoulli equation of MFs [4] for two points at the surface. In equilibrium, the sum of all contributions of pressure at point (1), close to the wire, is equal to the sum at point (2), far away from it. With the assumption that the surface $\xi(r)$ and the magnetic field $\mathbf{H}=I /(2 \pi r) \mathbf{e}_{\varphi}$ of the wire tend to zero as $r$ goes to infinity, the equation reads

$$
p_{0}^{(1)}+p_{c}^{(1)}+\rho g \xi^{(1)}-\frac{\mu_{0}}{2}\left[M_{\mathrm{n}}^{(1)}\right]^{2}-\mu_{0} \int_{0}^{H^{(1)}} M \mathrm{~d} H^{\prime}=p_{0}^{(2)}+p_{c}^{(2)}-\frac{\mu_{0}}{2}\left[M_{\mathrm{n}}^{(2)}\right]^{2},
$$

where $p_{0}$ is the hydrostatic pressure and $p_{c}=\sigma K$ is the capillary pressure with the surface tension $\sigma$ and the curvature $K$. The current through the wire is 

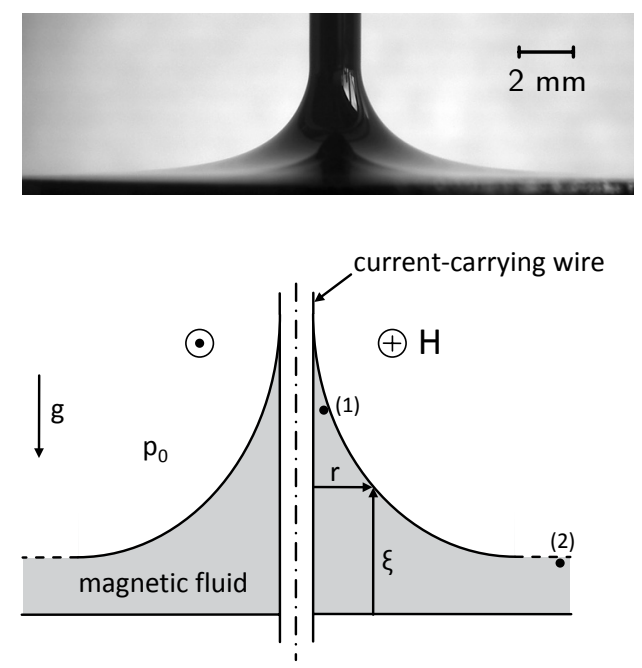

Fig. 1. (top) Meniscus shape of the magnetic fluid EMG 909 around the wire carrying a current of 70 A. Courtesy of K. May [6]. (bottom) Sketch of the setup (for details see the text).

denoted by $I$, the density of the fluid by $\rho$ and the acceleration due to gravity by $g$. The hydrostatic pressure is the same at both points and $p_{c}^{(2)}$ goes to zero due to $K^{(2)}=0$ with $\xi \rightarrow 0$ for $r \rightarrow \infty$. With a linear law of magnetization, $\mathbf{M}=\chi \mathbf{H}$, and the azimuthal property of the magnetic field, one finally yields an analytical equation for the free surface of the magnetic fluid [5]

$$
\rho g \xi-\sigma \frac{\xi^{\prime}+\xi^{\prime 3}+r \xi^{\prime \prime}}{\left(1+\xi^{\prime 2}\right)^{3 / 2}} \frac{1}{r}-\frac{\mu_{0} \chi I^{2}}{8 \pi^{2}} \frac{1}{r^{2}}=0
$$

where $\chi$ denotes the susceptibility of the MF and the primes denote the derivatives with respect to $r$.

An analytical solution for the simple case of $\sigma=0$ was already given in [4], but an analytical solution for the full problem (2) is not in sight. The reason is the nonlinear character of the equation caused by the curvature in the term of capillary pressure. Therefore, John et al. in [5] proposed a transformation, which regularizes the problem and allows a numerical solution. Nevertheless, Eq. (2) is an approximation since the derivation of the Bernoulli equation assumes isothermal conditions as well as an irrotational and stationary flow field. Therefore, the aim of this study is to present the numerical simulation of the underlying Navier-Stokes equation

$$
\rho\left[\frac{\partial \mathbf{v}}{\partial t}+(\mathbf{v} \operatorname{grad}) \mathbf{v}\right]=-\operatorname{grad} p+\eta \Delta \mathbf{v}+\rho \mathbf{g}+\mu_{0}(\mathbf{M} \operatorname{grad}) \mathbf{H},
$$

in two dimensions, while keeping the first assumption of isothermal conditions. Since the experiments were performed with the fluid EMG 909, we use the following standard set of material parameters: $\rho=0.20 \mathrm{~kg} / \mathrm{m}^{3}, \sigma=0.0258 \mathrm{~N} \mathrm{~m}^{-1}$ and $\chi=0.61[6]$. The question of the used dynamical viscosity is referred to Sec. 4 . In the two-dimensional $r-\xi$ coordinate system, the Kelvin force density is given by $\mathbf{f}_{\mathrm{K}}=\mu_{0}(\mathbf{M}$ grad $) \mathbf{H}=\mu_{0} M(\partial H / \partial r, 0)$, where $\mathbf{H} \| \mathbf{M}$ is used and $M(H)$ denotes the absolute value of the magnetization (magnetic field). For the final determination, either a linear law of magnetization, as given before, is used or the experimental data of the nonlinear behaviour of the magnetization (Fig. 2(top)). 
Meniscus of a magnetic fluid in the field of a current-carrying wire:

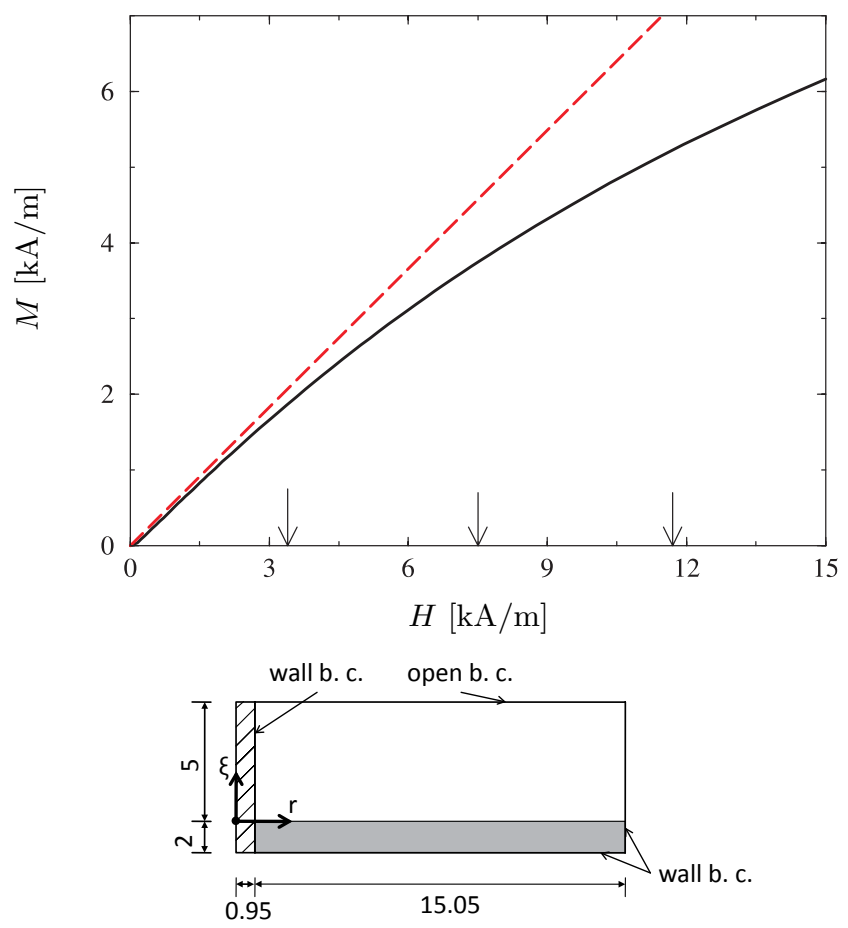

Fig. 2. (top) Experimentally measured magnetization curve (solid line) of EMG 909 in the relevant range of $H$. The dashed line indicates a linear law of magnetization with $\chi=0.61$. The vertical arrows indicate the strength of the magnetic field at $r=0.95 \mathrm{~mm}$ for $I=20 \mathrm{~A}, 45 \mathrm{~A}$, and $70 \mathrm{~A}$ (from left to right). (bottom) Geometry and boundary conditions (b. c.) of the used numerical region. The gray (shaded) region indicates the area of the magnetic fluid (wire). All measures are in millimeters.

3. Numerical methods. As a first step to tackle the problem, the numerics is done in two dimensions reflecting the axial symmetry of the problem. The numerical calculations are made with the commercial software package ANSYS FLUENT using the finite volume method for the two phases fluid and air. The domain of calculation is a rectangle with a width of $16 \mathrm{~mm}$ and a height of $7 \mathrm{~mm}$ according to the experimental setup in [6], where the origin is shifted by $2 \mathrm{~mm}$ in the vertical direction by the thickness of the layer of magnetic fluid, see Fig. 2(bottom). No-slip boundary conditions (wall b. c.) are imposed at all boundaries with the exception of the upper horizontal boundary, where a so-called open boundary condition is present. This condition allows an unconstrained flow of air in or out of the system. By using a finite volume method, the numerical results are affected by the so-called "flotsam" generated during the reconstruction of the phase boundary between the MF and air. The flotsam is considerably reduced by the use of FLUENT's GEO-Reconstruct method, which is based on Youngs' technique [7]. That technique is superior to other approaches as a comparing study [8] shows. Only in the column nearest to the wire a few cells of flotsam remain. Since beyond a simulation time of $t=2.5 \mathrm{~s}$ in all tested cases this last flotsam does not move any more and does not influence the shape of the surface, it was removed. The change of the total mass of MF due to that removal can be neglected.

The FLUENT's numerical method for modeling the surface tension is based on the continuum surface force (CSF) model proposed in [9]. The CSF model 


\section{P.-B. Eißmann, A. Lange, S. Odenbach}

uses the static angle of contact established when the fluid is at rest. In agreement with this definition, the angle of contact of the MF with the wire was measuredin [10]. Contrary, neither information about a change of this angle during the rise of the fluid at the wire nor any measurements of the angle of contact at the wall of the vessel are available [10]. Thus the conducted numerics utilizes static angles of contact only, where one of them is experimentally known.

4. Results and discussion. As a first parameter, the influence of the dynamic viscosity on the surface is studied since in the Navier-Stokes equation the term of viscous friction appears. Beside the value of $\eta=6 \cdot 10^{-3} \mathrm{~kg} /(\mathrm{m} \mathrm{s})$ taken from $[11,12]$, a considerable lower (higher) value of $\eta=1 \cdot 10^{-3} \mathrm{~kg} /(\mathrm{m} \mathrm{s})$ $\left(\eta=10 \cdot 10^{-3} \mathrm{~kg} /(\mathrm{m} \mathrm{s})\right)$ is considered in Fig. 3. In all three cases, the final shape of the surface is identical, which confirms the use of the Bernoulli equation of a quiescent MF. The numerics approves that the viscosity of the fluid does not play a role in the determination of the final surface. That is why for all remaining calculations $\eta=1 \cdot 10^{-3} \mathrm{~kg} /(\mathrm{m} \mathrm{s})$ is set.

The next parameter, which is tested, is the angle of contact $\phi_{1}$ of the fluid at the wire. In [10] it was shown that a change of this angle plays a minor role in shaping the surface. This experimental result is confirmed by our numerical data as long as $\phi_{1}$ is not changed too much. A doubling or a bisection of the reference value $\phi_{1}=8.7^{\circ}[10]$ causes practically no variation of $\xi(r)$ over the entire range of $r$, see Fig. 4(a). But a larger variation to $\phi_{1}=45^{\circ}$ or $\phi_{1}=90^{\circ}$ can trigger major changes, see Fig. 4(b). At the wire the height of the surface drops to the half $\left(\phi_{1}=45^{\circ}\right)$ or vanishes nearly completely $\left(\phi_{1}=90^{\circ}\right)$. Complementary, the thickness of the layer of MF increases for $r \geq 4 \mathrm{~mm}$.

The other angle of contact is $\phi_{2}$ at the rim of the vessel. That angle was not measured and set to $\phi_{2}=90^{\circ}$ in the calculations in $[5,6]$. The numerics offers now the possibility to analyse the influence of this angle, too. Already a moderate reduction to $\phi_{2}=70^{\circ}$ or $\phi_{2}=50^{\circ}$ changes nearly the entire shape, see

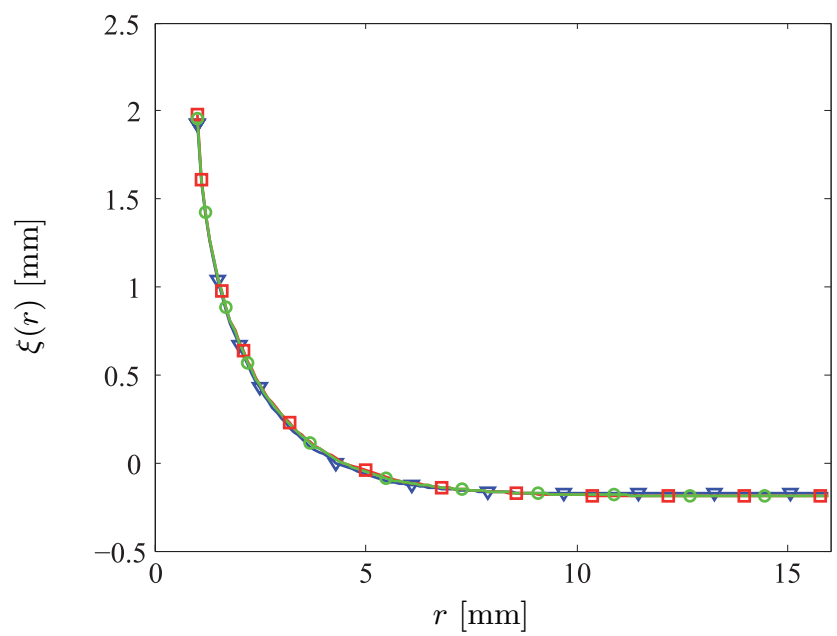

Fig. 3. (color online) The surface $\xi(r)$ versus the distance $r$ from the wire as a function of the viscosity of the MF: $\eta=1 \cdot 10^{-3} \mathrm{~kg} /(\mathrm{ms})$ (blue line and triangles), $\eta=6 \cdot 10^{-3} \mathrm{~kg} /(\mathrm{m} \mathrm{s})$ (red line and squares) and $\eta=10 \cdot 10^{-3} \mathrm{~kg} /(\mathrm{m} \mathrm{s})$ (green line and circles). The shape of the surface is identical in all three cases. The angle of contact at the wire is $\phi_{1}=8.7^{\circ}[10]$, at the rim of the vessel the angle is set to $\phi_{2}=90^{\circ}$. A current of $I=20 \mathrm{~A}$ and the experimentally measured magnetization curve (Fig. 2(top)) is used. 
Meniscus of a magnetic fluid in the field of a current-carrying wire:

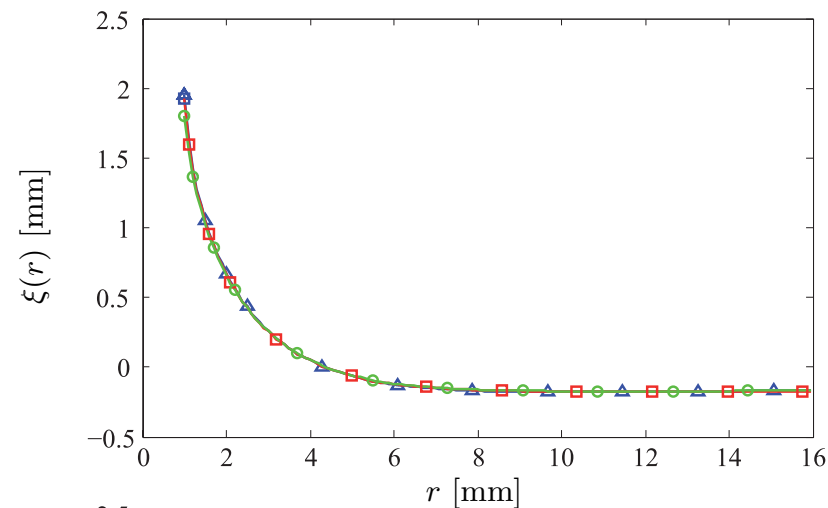

(a)

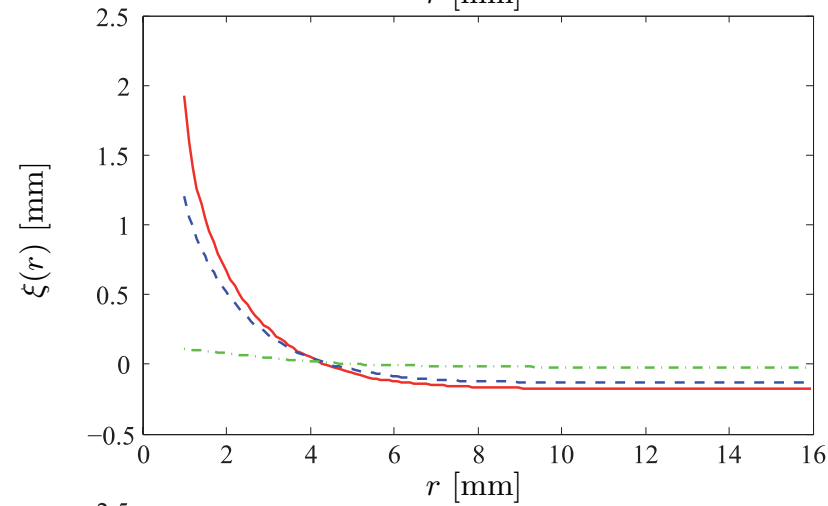

(b)

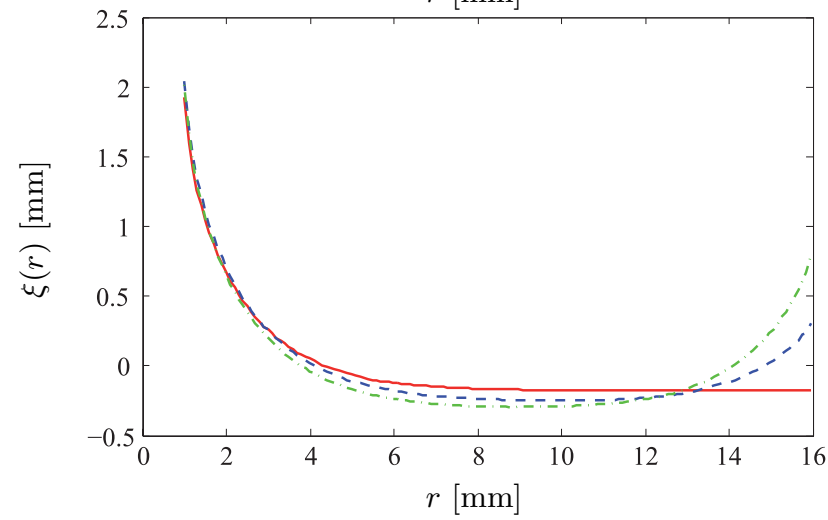

$(c)$

Fig. 4. (color online) The surface $\xi(r)$ versus the distance $r$ from the wire as a function of the angle of contact $(a),(b)$ at the wire, and $(c)$ at the rim. $(a) \phi_{1}=4.3^{\circ}$ (blue line and triangles), $\phi_{1}=8.7^{\circ}$ (red line and squares) and $\phi_{1}=17.4^{\circ}$ (green line and circles). Only very close to the wire small changes are detectable. (b) $\phi_{2}=8.7^{\circ}$ (red solid line), $\phi_{2}=45^{\circ}$ (blue dashed line) and $\phi_{2}=90^{\circ}$ (green dash-dotted line). With an increasing of $\phi_{2}$, a drop of the height at the wire and an increase of the thickness of the fluid layer away from the wire are observable. (c) $\phi_{2}=90^{\circ}$ (red solid line), $\phi_{2}=70^{\circ}$ (blue dashed line), and $\phi_{2}=50^{\circ}$ (green dash-dotted line). With a lowering of $\phi_{2}$, an increase of the height at the rim and a decrease of the thickness of the fluid layer over a wide rage of $r$ between $2 \mathrm{~mm}$ and $12 \mathrm{~mm}$ is detectable. The remaining parameters are those of Fig. 3 .

Fig. 4(c). There is a notable increase of $\xi$ in a small outer range and a lowering of the layer thickness over most of the remaining range of $r$. Only the immediate neighbourhood of the wire is not affected. 


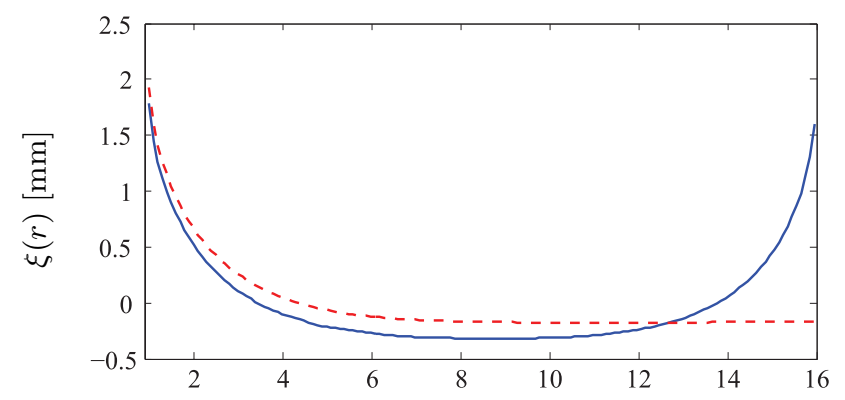

(a)

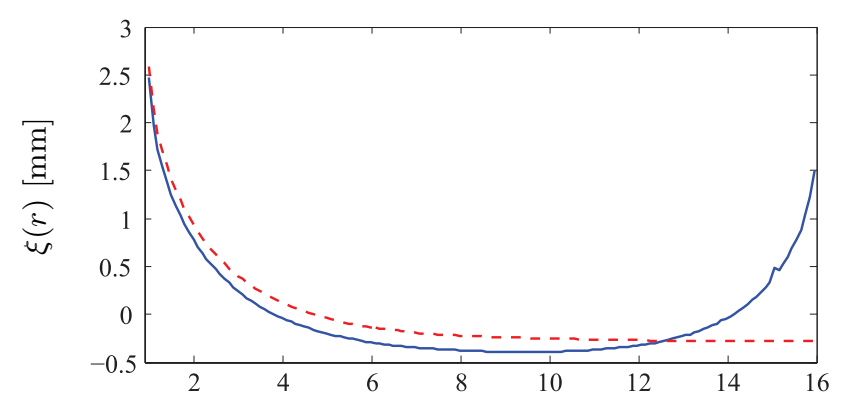

(b)

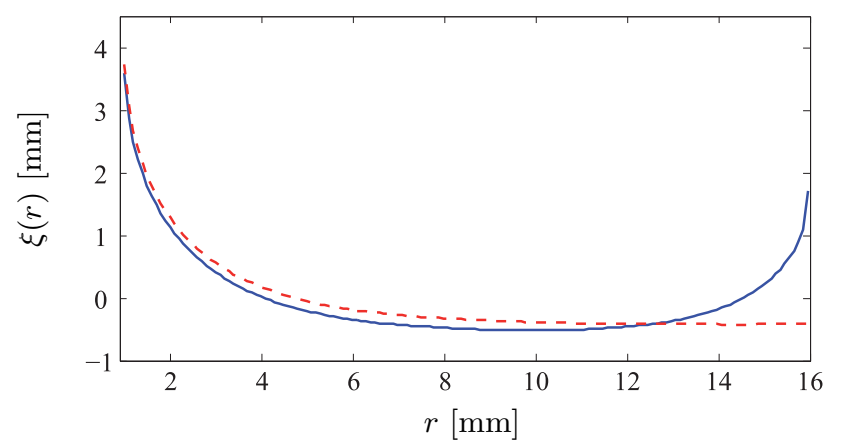

$(c)$

Fig. 5. (color online) The surface $\xi(r)$ (blue solid line) versus the distance $r$ from the wire as a function of current with the condition $\phi_{1}=\phi_{2}$ : (a) $I=20 \mathrm{~A}$ and $\phi_{1}=8.7^{\circ}$, (b) $I=45 \mathrm{~A}$ and $\phi_{1}=6.3^{\circ}$, and $(c) I=70 \mathrm{~A}$ and $\phi_{1}=3.4^{\circ}$. The red dashed line presents the surface for $\phi_{2}=90^{\circ}$ for comparison. The remaining parameters are those of Fig. 3.

In a second analysis concerning the angles of contact, the surface $\xi(r)$ is determined, when both angles are the same, but the strength of the current is increased. Fig. 5(a) shows the results for $I=20 \mathrm{~A}$ and $\phi_{1}=\phi_{2}=8.7^{\circ}$, part $(b)$ for $I=45 \mathrm{~A}$ and $\phi_{1}=\phi_{2}=6.3^{\circ}$ and part $(c)$ for $I=70 \mathrm{~A}$ and $\phi_{1}=\phi_{2}=3.4^{\circ}$. The angles of contact for the higher currents are taken from [10]. As in the analysis before, the general behaviour can be found here again for all three strengths of the current. The shape of the surface is not affected only in the adjacent region of the wire. The thickness of the layer shrinks over most of the range of $r$ compared to the thickness for $\phi_{2}=90^{\circ}$. Concerning the rise of the surface at the rim, a tendency can be observed: the lower the angle of contact at the rim, from $(a)$ to (c), the higher the rise of $\xi$ at the edge of the vessel.

A consequence of the calculations is that a measurement of the angle of contact at the rim would be desirable to improve the comparison between numerics and experiment. 
Meniscus of a magnetic fluid in the field of a current-carrying wire:
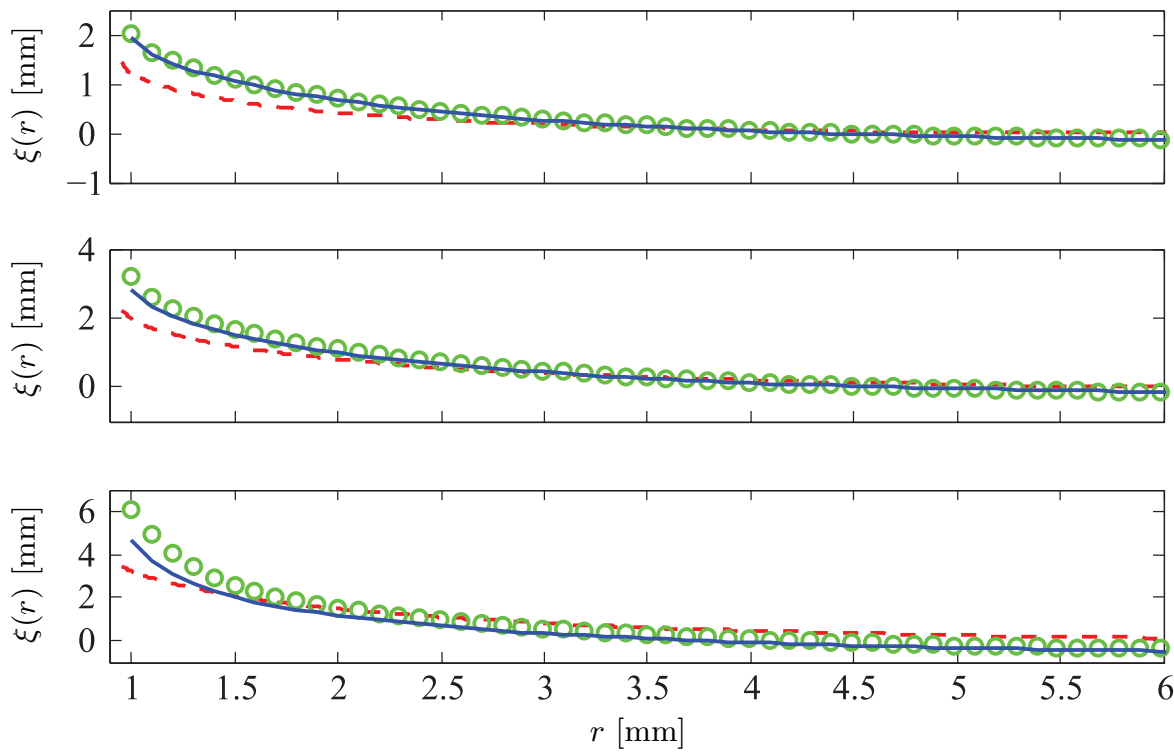

Fig. 6. (color online) The surface $\xi(r)$ versus the distance $r$ from the wire as a function of the strength of the currect: (a) $I=20 \mathrm{~A}(H \simeq 3.4 \mathrm{kA} / \mathrm{m}),(b) I=45 \mathrm{~A}$ $(H \simeq 7.5 \mathrm{kA} / \mathrm{m}),(c) I=70 \mathrm{~A}(H \simeq 11.7 \mathrm{kA} / \mathrm{m})$. The red dashed line shows the measured profiles, the blue solid line (green circles) the calculated ones for a linear magnetization with $\chi=0.61(\chi=0.8)$. For details see the text.

After testing the influence of the different angles of contact and the viscosity on the shape of the surface, the measured profiles for different strengths of the current $I[10]$ will be now compared to the calculated ones, where $\eta=1 \cdot 10^{-3} \mathrm{~kg} /(\mathrm{ms})$, $\phi_{1}=8.7^{\circ}$ and $\phi_{2}=90^{\circ}$ are fixed. Beside the current, the strength of the corresponding magnetic field at the radius $r=0.95 \mathrm{~mm}$ of the wire will be given since $H$ is the essential quantity. Fig. 6 presents the profiles for a linear law of magnetization with $\chi=0.61$. For all three strengths one can see that close to the wire the calculated height is above the measured one, the difference is about $1 \mathrm{~mm}$. Further away from the wire, both profiles approach each other with the tendency that the calculated profiles underestimate the measured ones, which is most pronounced for the highest values of the magnetic field, see Fig. 6(c). It is a direct consequence of the overestimation of $\xi$ close to the wire combined with the conservation of mass. Additionally, the susceptibility of $\chi=0.8$ given by [12] for EMG 909 is tested (green symbols in Fig. 6). For low and moderate fields (see Fig. 6(a,b)), there is hardly any difference detectable, but for a high field the concave meniscus extends to larger $r$ values (see Fig. 6(c)). The reason is an apparent higher magnetization for the larger value of $\chi$ at high fields.

For the largest tested value of $I$, the relevant magnetic fields lie in the nonlinear regime of magnetization of EMG 909, see Fig. 2(top). That is why the nonlinear magnetization was taken into account in the calculations. The corresponding results are shown in Fig. 7. The differences in both calculated profiles (compare blue solid lines and green symbols in Fig. 7) become more clearly as $H$ increases since the difference between the linear and nonlinear law of magnetization increases, too. Therefore, the mismatch between the calculated and the measured profile for low fields at a current of $I=20 \mathrm{~A}$ in the vicinity of the wire remains. For moderate fields at $I=45 \mathrm{~A}$, the difference shrinks, whereas for high fields at $I=70 \mathrm{~A}$ a satisfying agreement between experiment and numeric is found. 

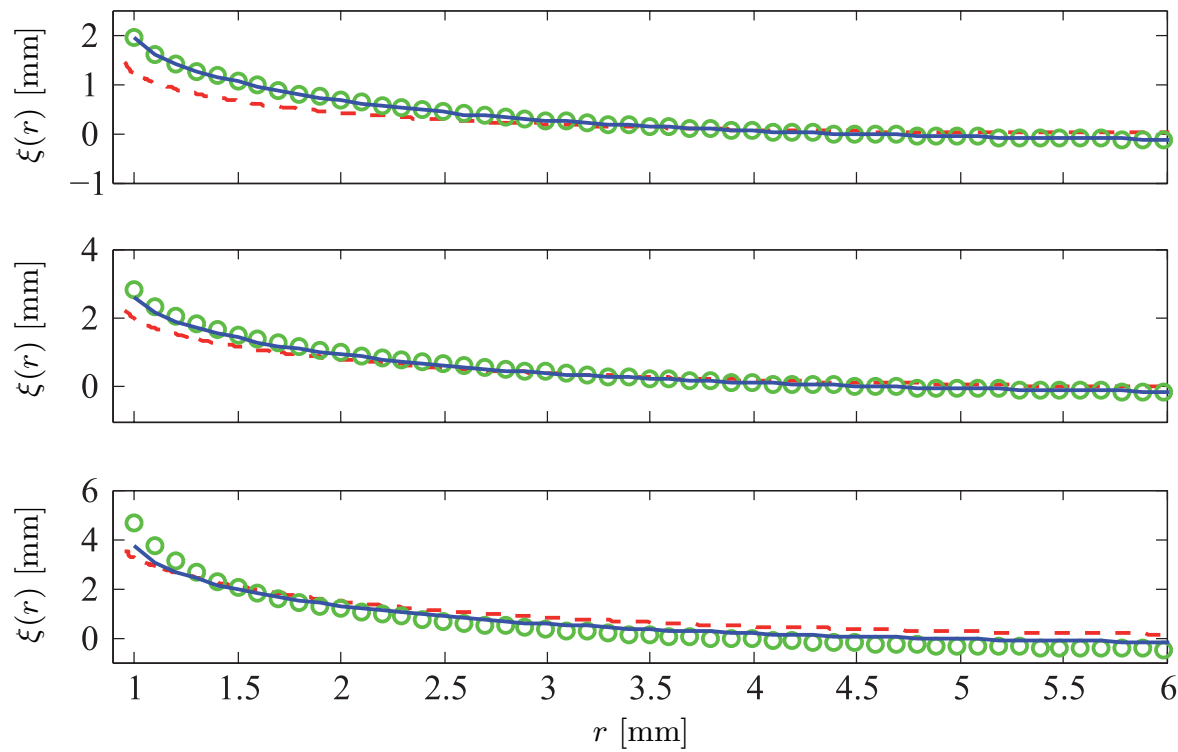

Fig. 7. (color online) The surface $\xi(r)$ versus the distance $r$ from the wire as a function of the strengthof the currect: (a) $I=20 \mathrm{~A}(H \simeq 3.4 \mathrm{kA} / \mathrm{m}),(b) I=45 \mathrm{~A}$ $(H \simeq 7.5 \mathrm{kA} / \mathrm{m}),(c) I=70 \mathrm{~A}(H \simeq 11.7 \mathrm{kA} / \mathrm{m})$. The red dashed line shows the measured profiles, the blue solid line (green circles) shows the calculated ones for a nonlinear magnetization (a linear magnetization with $\chi=0.61$ ). For details see the text.

As causes for the discrepancies at low fields, different reasons might come into consideration. The overall error of $0.2 \mathrm{~mm}$ in the measurements of the height [10] mounts to a relative error of $14 \%$ for $\xi$ at the wire with the lowest tested field of $H \simeq 3.4 \mathrm{kA} / \mathrm{m}$. Contrary, the relative error reduces to less than $6 \%$ at the highest tested field of $H \simeq 11.7 \mathrm{kA} / \mathrm{m}$. Therefore, the measured profiles at low fields are more sensitive to the preparation of the experimental setup. Particularly, a possible influence of the fact that the wire was wetted is difficult to assess. Since for low fields the influence of the surface tension and curvature dominates the shaping of the surface, both quantities have to be used according to their genuine status. The surface tension was determined by the capillary rise method [6], which is very accurate for wetting fluids as the used magnetic fluid EMG 909. But a genuine description of the real three-dimensional surface is not given by a twodimensional modeling as in the presented study here. Therefore, it is assumed that this is the main reason for the discrepancies and that three-dimensional numerical calculations will likely overcome them at low fields.

5. Summary. In summary, the presented two-dimensional calculations show that the viscosity of the magnetic fluid has no influence on the final shape of the meniscus around a current-carrying vertical and cylindrical wire. Contrary, larger changes of the angle of contact at the wire as well as moderate modifications of the angle of contact at the rim of the vessel can influence the meniscus over the entire vessel. Particularly, the latter result supports a measurement of this angle, so far missing in the experiment, for a more profound comparison between numerical and experimental results. For a linear law of magnetization and for low as well as for moderate strengths of the current, the profiles agree satisfyingly up to $2 \mathrm{~mm}$ to the wire. Closer to the wire and for high values of the current, the agreement is not satisfying. Therefore, a nonlinear law of magnetization was taken into account. 
Meniscus of a magnetic fluid in the field of a current-carrying wire:

It causes an improvement between numerics and experiment only at the highest tested values of the current, but disagreements close to the wire remain.

Based on these results, a better agreement with the experiment is expected for a three-dimensional geometry, where additionally the curvature of the surface with respect the azimuthal angle can be described. Furthermore the assumption of isothermal conditions and possible influences on the material parameters, in particular, on the surface tension, will be presented and discussed in a forthcoming publication.

Acknowledgements. We would like to express our gratitude to Kathrin Mayfor providing the material data of EMG 909 and the measured profiles of the surface. Also the authors acknowledge Thomas John for clarifying discussions.

\section{REFERENCES}

[1] R. Richter AND A. LANGe. Surface instabilites of ferrofluids. In S. OdenBACH, editor, Collodial magnetic fluids: bacisc, development and application of ferrofluids, vol. 763 of Lecture Notes in Physics (Springer Verlag, Berlin, 2009) .

[2] C. Chen And C. Li. Ordered microdroplet formations of thin ferrofluid layer breakups. Phys. Fluids, vol. 22 (2010), no. 1, p. 014105.

[3] S. Kodama. Dynamic ferrofluid sculpture: organic shape-changing art forms. Commun. ACM, vol. 51 (2008), no. 6, pp. 79-81.

[4] R.E. Rosensweig. Ferrohydrodynamics (Cambridge University Press, Cambridge, 1985).

[5] T. John, D. Rannacher, And A. Engel. Influence of surface tension of the conical meniscus of a magnetic fluid in the field of a current-carrying wire. $J$. Magn. Magn. Mat., vol. 307 (2007), no. 1, pp. 31-35.

[6] T. John, M. May, and R. Stannarius. Meniscus of a ferrofluid around a vertical cylindrical wire carrying electrical current. Phys. Rev. E, vol. 83 (2011), no. 5, p. 056308.

[7] D.L. Youngs. Time-dependent multi-material flow with large fluid distortion. In K. W. Morton And M. J. Baines, editors, Numerical methods for fluid dynamics (Academic, New York, 1982) pp. 273-285.

[8] M. Rudman. Volume-tracking methods for interfacial flow calculations. Int. J. Numer. Methods Fluids, vol. 24 (1997), no. 7, pp. 671-691.

[9] J.U. Brackbill, D.B. Kothe, and C. Zemach. A continuum method for modeling surface tension. J. Comput. Phys., vol. 100 (1992), no. 2, pp. 335354.

[10] K. MAY. Untersuchung des Meniskus von Ferrofluid an einem stromdurchflossenen Draht (Tech. rep., Univ. Magdeburg, 2010).

[11] T. Mahr and I. Rehberg. Nonlinear dynamics of a single ferrofluid-peak in an oscillating magnetic field. Physica D, vol. 111 (1998), no. 1-4, pp. 335-346.

[12] Ferrotec Corp. www.ferrotec-europe.de/en/htmls/fluid.data.php.

Received 08.06.2011 